\title{
Hemispheric Differences in Self-evaluation Errors of Upper Extremity Movement in Patients with Chronic Stroke
}

\author{
Sola Choi ${ }^{1,2}$, Jongim Won ${ }^{1}$, Kyuenam Park ${ }^{1}$ and Sujin Kim ${ }^{1, *}$ (D) \\ ${ }^{1}$ Department of Physical Therapy, Jeonju University, Jeonju, South Korea \\ ${ }^{2}$ Department of Physical and Rehabilitation Medicine, Samsung Hospital, Seoul, South Korea
}

(Received February 18, 2021; Final revision November 22, 2021; Accepted December 7, 2021; FiRst Published OnLine January 24, 2022)

\begin{abstract}
Objective: There is growing evidence that the side of brain lesions results in distinct upper extremity deficits in motor control, movement behavior, and emotional and cognitive function poststroke. We investigated self-evaluation errors, which are the differences in scores between patient self-evaluation and clinician evaluations, and compared patients with left hemisphere damage (LHD) and right hemisphere damage (RHD) poststroke. Method: Twenty-eight patients with chronic stroke $(\mathrm{LHD}=16)$ performed the actual amount of the test twice with a one-week interval. We videotaped the participants' movements, and participants with stroke and evaluators graded the quality of movement scores by watching video recordings. Results: Self-evaluation errors were significantly lower in patients with LHD than in those with RHD $(t=2.350, p=.019)$. Interestingly, this error did not change after the clinician provided the correct score as feedback. Chi-squared analysis revealed that more patients with LHD underestimated their movements $\left(\chi^{2}=9.049, p=.002\right)$, while more patients with RHD overestimated $\left(\chi^{2}=7.429, p=.006\right)$ in the send evaluation. Furthermore, there were no correlations between self-evaluation error and age, cognitive function, physical impairment, ability to control emotions, or onset months poststroke. Conclusions: Patients with stroke and therapists evaluated the same movements differently, and this can be dependent on hemispheric damage. Therapists might need to encourage patients with LHD who underestimate their movement to ensure continuous use of their more-affected arm. Patients with RHD who overestimate their movement might need treatment to overcome impaired self-awareness, such as video recordings, to protect from unexpected dangerous situations.
\end{abstract}

Keywords: Arms, Hemispheric specialization, Performance, Self-evaluation, Strokes, Upper extremity paresis, Video recording

\section{INTRODUCTION}

Impaired self-awareness, known as anosognosia or unawareness, is a disorder in which patients with brain damage fail to recognize the severity of deficits in motor, sensory, and cognition function (Hartman-Maier, Soroker, Ring \& Katz, 2002; Orfei et al., 2007; Pia et al., 2013; Prigatano, 2005). Unawareness has been extensively studied in patients with traumatic brain injury (Fischer, Gauggel \& Trexler, 2004; Prigatano, 1996; Sherer, Hart \& Nick, 2003), and its scope has been extended to the stroke population. (Cocchini, Beschin \& Della Sala, 2018; D'Imperio, Bulgarelli, Bertagnoli, Avesani \& Moro, 2017; Fotopoulou, Pernigo, Maeda, Rudd \& Kopelman, 2010; Hartman-Maeir, Soroker \& Katz, 2001; Jenkinson, Preston \& Ellis, 2011; Pia,

*Correspondence and reprint requests to: Sujin Kim, Department of Physical Therapy, Jeonju University, 303 Cheonjam-ro, Jeonju-si, Jeollabuk-do, South Korea. E-mail: sujink@jj.ac.kr
Neppi-Modona, Ricci \& Berti, 2004; Spinazzola, Pia, Folegatti, Marchetti \& Berti, 2008). It is commonly reported that the frequency and severity of unawareness are higher in patients with right hemisphere damage (RHD) than with left hemisphere damage (LHD) (Nurmi Laihosalo \& Jehkonen, 2014; Orfei et al., 1973; Pia et al., 2004). Additionally, the occurrence of unawareness is more frequent in the acute state (Starkstein, Jorge \& Robinson, 2010). However, a significantly higher percentage $(40 \%)$ of patients with LHD show unawareness when the appropriate assessment tool is used (Cocchini, Beschin, Cameron, Fotopoulou \& Della Sala, 2009), and the unawareness remains beyond the acute phase (Jehkonen, Laihosalo \& Kettunen, 2006; Orfei et al., 1973). Because unawareness is multi-faceted, we need to explicitly or implicitly assess subtypes of unawareness using various measurement methods in the multiple domains (Cocchini, Beschin, Fotopoulou \& Della Sala, 2010; Marcel, Tegnér \& Nimmo-Smith, 2004), especially for the patients in the chronic stroke stage. 
Here, we extend our understanding of unawareness by exploring the differences between chronic patients with RHD and LHD in evaluating task performance based on video recordings. Recent studies have shown that the evaluation of self-awareness is more accurate when using a video recording, in which the participants evaluate the movement after executing the actual task or answer questions from the third-person perspective (Fotopoulou, Rudd, Holmes \& Kopelman, 2009; Marcel et al., 2004). However, these studies have reported the effectiveness of video feedback on a single case of stroke or patients in acute settings; research with a larger group of patients with chronic stroke to assess unawareness via video-based self-observation is needed.

The frequency of spontaneous use of the more-affected limb during daily activities is higher in the patients with LHD than those with RHD, especially for individuals with a premorbid dominant right hand (Haaland et al., 2012; Kim, Park, Han, Winstein \& Schweighofer, 2018; Mani, Przybyla, Good, Haaland \& Sainburg, 2014). This increased arm use can be viewed as a 'self-training' and leads to motor performance enhancement (Hidaka, Han, Wolf, Winstein \& Schweighofer, 2012). We hypothesized that increased arm use can also influence self-awareness due to the positive or negative experiences using the more-affected arm over time (Taub, Uswatte \& Elbert, 2002). For example, patients with LHD who often use the more-affected arm may be more likely to underestimate their performance if they have more unsuccessful experiences. One recent study showed that patients with LHD following acute-to-subacute stroke tend to underestimate their ability during upper extremity movement (Fowler, Della Sala, Hart \& McIntosh, 2018). In contrast, the patients with RHD tend to overestimate (Fowler et al., 1973), and a similar trend is found in both stroke (Marcel et al., 2004) and traumatic brain injury populations (Mizuno, 1991; Prigatano, 1996). The association between spontaneous use of the more-affected arm and self-evaluation based on video recording in patients with chronic stroke could provide further understanding of the nature of unawareness.

This study aimed to investigate whether patients with LHD and RHD following stroke showed a distinct capability for self-awareness of their upper extremity (UE) movement if they watched a video of their movement. Additionally, we sought to understand whether self-evaluation could be corrected in patients with chronic stroke if the clinician provided feedback. Finally, the relationships between unawareness and spontaneous use and between unawareness and impairment were explored. We hypothesized that there is a difference in self and clinical evaluation in patients with hemiparesis following stroke and that patients with LHD or RHD under- or overestimate the more-affected arm movement, respectively.

\section{METHOD}

\section{Participants}

Twenty-eight patients with unilateral stroke (LHD, $n=16$; RHD, $n=12$ ) participated in this study. Participants with stroke were recruited from seven rehabilitation centers in South Korea. All participants had traditional physical and occupational therapy, on average, $40 \mathrm{~min}$ each, and they did not participate in other research interventions. The inclusion criteria were: (1) clinical diagnosis of a first or recurrent unilateral ischemic or hemorrhagic stroke in the chronic stage (at least 3 months after stroke onset) (Nakase, Yoshioka \& Suzuki, 2011); (2) age $\geq 21$ years and impaired UE motor function, as indicated by the Fugl-Meyer upper extremity scale (FM-UE, $\geq 19$ out of 66); (3) no unilateral sensory and motor neglect, as determined by line cancelation in the Albert test (Albert, 1973; Blumenfeld, 2002); (4) understanding and following the instructions of the experimenter (Fowler et al., 2018). Patients with a history of recent surgeries, pain, or orthopedic injuries affecting the movement of UE, or with severe cognitive deficits (MMSE < 18) (Lee, Cheong, Oh \& Hong, 2009), communication problem (SIS - communication subscale $<3.5$ ), and severe spasticity were excluded. All participants with stroke were right-handed before the stroke, as measured using the Edinburgh Handedness Inventory (EHI). Their memory, mood, communication, and activities of daily livings were measured using the Stroke Impact Scale (SIS) (Duncan, Bode, Lai \& Perera, 2003). The Ethics Committee of Jeonju University approved the study protocol (jjIRB-171115-HR-2017-1109), and all participants provided written informed consent for participation. They also signed an agreement to permit videotaping for the entire experiment. This study was conducted in accordance with the Declaration of Helsinki.

Table 1 summarizes the general characteristics of the participants with stroke. All participants with stroke were in either early chronic phase (3-6 months, four participants in each group) or late chronic phase ( $>6$ months), and they had mild-to-moderate impairment on their UE (median score of FM-UE: 42 vs. 49 for LHD and RHD, respectively). There were no significant differences in age $(t=.112, p=.912)$, cognitive function (Mini-Mental State Examination, $W=97, p=.160)$, time since stroke onset $(W=83, p=.551)$, degree of hand preference (EHI, $W=54, p=.120$ ), and visuomotor neglect between the LHD and RHD groups (Albert's test, $W=67.5, p=.722$ ). In addition, the two groups did not differ in the physical impairments measured by the arm, wrist, and hand sub-items of FM (all variables, $p>.05)$. Memory, mood, communication, ADLs, and hand function (measured by SIS) did not differ between the groups (all variables, $p>.05$ ).

\section{Clinical Measurements}

\section{Actual amount of use test assessing the self-evaluation error}

The actual amount of use test (AAUT) was developed by Taub et al. to measure an individual's spontaneous use of the more-affected limb during ADL, for example, opening a file folder, picking up a photo album, or turning the pages of a photo album (Taub \& Uswatte, 2004; 
Table 1. General characteristics of the participants $(N=28)$

\begin{tabular}{lcc}
\hline \hline Variable & LHD $^{\mathrm{b}}$ & RHD $^{\mathrm{c}}$ \\
\hline Number & 16 & 12 \\
Age (years) & $64.0(58.0-69.3)^{\mathrm{a}}$ & $69.0(61.0-75.0)$ \\
Sex (\% male) & $81.25 \%$ & $46.67 \%$ \\
Time since onset (month) & $16.5(8.0-28.3)$ & $12.0(5.0-22.0)$ \\
EHI $(100$ maximum) & $100(97.5-100)$ & $100(100-100)$ \\
MMSE $^{\mathrm{e}}$ (30 maximum) & $27.5(26.8-30)$ & $24.4(23.5-27.5)$ \\
Albert's test (39 maximum) & $38.8(39-39)$ & $38.8(39-39)$ \\
FM-UE & & $49(35.3-55.5)$ \\
$\quad$ Whole score (66 maximum) & $42(35.5-51.0)$ & $24.5(16.5-28.3)$ \\
Upper arm & $22.1(17.3-27.3)$ & $8.5(6.0-9.3)$ \\
$\quad$ Wrist & $7.50(2.3-9.0)$ & $13(11.0-14.0)$ \\
Hand & $10.8(9.0-13.0)$ & $4.3(4.1-4.5)$ \\
Stroke impact scale & & $3.7(3.0-3.9)$ \\
$\quad$ Memory (5 maximum) & $4.4(4.0-4.9)$ & $4.6(3.7-5.0)$ \\
Mood & $3.5(3.0-3.5)$ & $3.9(3.1-4.5)$ \\
Communication & $4.5(4.0-4.7)$ & $3.0(2.0-4.3)$ \\
$\quad$ Activity of daily livings & $3.8(3.4-4.7)$ & \\
Hand & $1.9(1.3-3.7)$ & \\
\hline \hline
\end{tabular}

Uswatte \& Taub, 1999). The AAUT contains 17 items; however, we only used the first 14 items because the last three items are related to general, unpurposeful movements, for example, gesturing or posture (Han et al., 2013; Kim et al., 2018). We conducted AAUT in two ways: (1) a spontaneous use condition (sAAUT), in which the participants did not notice that they were undertaking the AAUT. We used a customized scenario that prompted the use of the moreaffected arm within our experimental environment; therefore, the participant naturally chose this arm to complete the tasks; (2) a forced use condition (fAAUT), in which participants were asked to use their more-affected arm (Han et al., 1973; Sterr, Freivogel \& Schmalohr, 2002). A video camera recorded the entire experimental process, and the recording was used to score the AAUT.

Since participants with stroke noticed the AAUT test after the forced use condition, they performed sAAUT only once before the fAAUT on the first visit day to prevent a behavioral bias (Figure 1). Next, patients performed the fAAUT twice repeatedly across testing days (fAAUT1, test; fAAUT2, retest). The fAAUT scoring was completed by patients and a well-trained and standardized clinician. The sAAUT scoring was performed by a clinician. The quality of movement (QOM) scale was used to evaluate movements, ranging from 0 to 5 , with $0=$ participants unable to move the moreaffected arm to complete the activity, and $5=$ normal performance as before stroke. Scoring included 0.5 units, for example, $0.5,1.5,2.5,3.5$, and 4.5 . for a more specific evaluation.

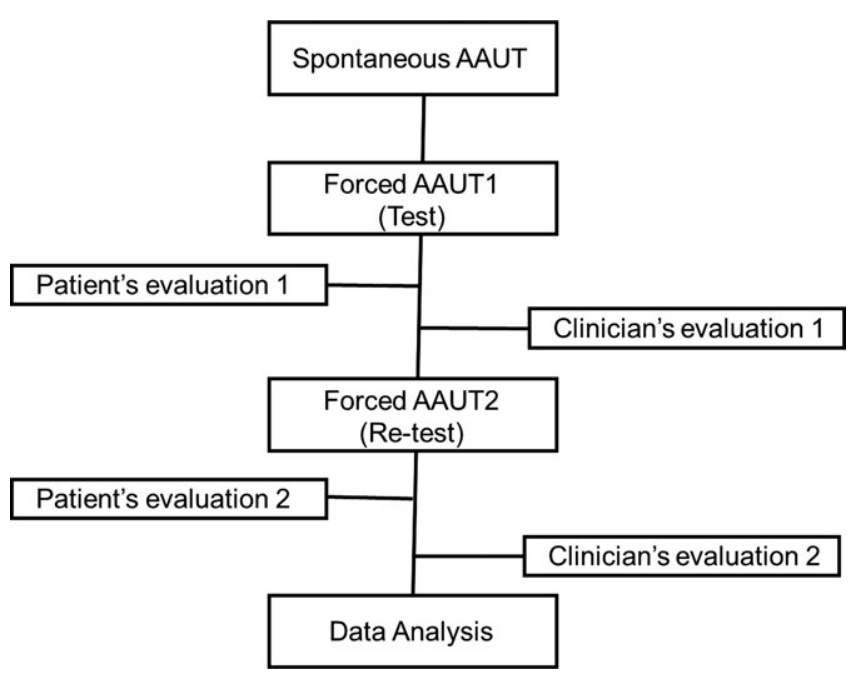

Fig. 1. Experimental procedure.

The difference between a patient's self-evaluation and the clinician's evaluation of the fAAUT was defined as the selfevaluation error. This had a potential range of -5 to +5 , with zero representing total agreement, and positive and negative scores representing overestimation and underestimation of the more-affected arm when performing tasks, respectively. Cut-off scores of +0.22 and -0.22 , calculated by the mean discrepancy between the fAAUT1 and fAAUT2 of the clinician's scores plus or minus two standard deviations, were used to determine the range for correct estimation. This cut-off 
allowed for a small proportion of evaluation errors that would occur in the self-awareness judgments of a human being (Della Sala, Cocchini, Beschin \& Cameron, 2009). A mean selfevaluation error greater or smaller than these cut-off scores indicated an over- or underestimation of UE performance, respectively (Fowler et al., 2018). The mean score between the cut-off scores was defined as the correct estimation.

\section{Impairment and use of the more-affected arm}

The FM-UE was developed to measure the severity of a patient's disability and the outcomes of medical rehabilitation. The maximum FM score was 66, indicating a normal motor ability (Fugl-Meyer, Jääskö, Leyman, Olsson \& Steglind, 1975). FM showed the excellent intra- and interrater reliability (ICC > .98) (See et al., 2013). The Actual spontaneous use of the more-affected arm was measured using sAAUT described above. A trained evaluator scored how well the subjects used their more-affected arm and hand by watching the videotape. Lower and higher scores indicated less and more use of the more-affected arm, respectively.

\section{Procedure}

Participants with stroke underwent two days of experimental evaluation with a one-week interval between evaluations to prevent any AAUT learning effect. On the first day, the experimenter explained the purpose of the study and obtained informed consent before the experiment started. First, the participants explicitly underwent FM-UE and SIS tests, and each item in the AAUT was naturally mixed with these tests via a customized scenario. Therefore, participants were unaware of the sAAUT. Immediately after sAAUT, the participants were instructed to repeat the same activity (fAAUT1, test). A trained and standardized evaluator graded the actual movement by watching the video (clinician's evaluation 1; Figure 1).

On the second day, participants viewed their actual movement via video recordings and assigned their QOM scores (patient's self-evaluation 1). Immediately after this, the participants were informed of the clinician's score so that they could compare the scores. Next, the participants performed the fAAUT (fAAUT2, retest) and were instructed to rate their actual performance (patient's self-evaluation 2). Once the participants had completed their self-evaluation, the clinician scored the second fAAUT (clinician's evaluation 2). Self-evaluation errors 1 and 2 were calculated as the patient's self-evaluation 1 or 2 minus the clinician's evaluation 1 or 2 , respectively.

\section{Statistical Analysis}

The Shapiro-Wilk test was used to assess the normal distribution of all data. A one-sample $t$-test was used to identify the self-evaluation error and a paired $t$-test was used to detect testretest differences in the evaluation of patients, clinicians, and self-evaluation errors. A linear mixed-effects analysis with categorical variables was used to assess the differences in self-evaluation between tests (first and second evaluation) and groups (LHD and RHD). As fixed effects, we entered the test and group into the model and set each subject as a random effect. First, we started with the model with a random intercept and sequentially extended models by adding groups, tests, and the interaction between groups and tests. Log-likelihood ratio tests (if the models were nested) or Akaike Inclusion Criterion (AIC; if the models were not nested) were used for model comparison. Next, a visual inspection of residuals versus fits plots and Q-Q plots was performed to check the residuals for normality and the presence of outliers (Lang et al., 2016; Varghese \& Winstein, 2020). There were no severe outliers, and departures from normality were mild. In addition, effect size was computed using partial omega square, with $0.01,0.06$, and 0.14 considered as small, medium, and large effect sizes, respectively. The chi-squared test was used to detect within-group differences in over-, under-, and correct estimations. Associations between the self-evaluation errors and other characteristic variables, including age, stroke onset, emotional control, MMSE score, and FM-UE score, were examined using Pearson or Spearman's rank correlation coefficients, depending on the data distribution. Statistical significance was set at .05 , and all results were reported as mean \pm standard deviation (SD) or median. All statistical analyses were performed using the statistical package version 3.6.2 (Team R Development Core, 2018).

\section{RESULTS}

\section{Patients and Clinicians Differently Evaluate Upper Extremity Movement}

The QOM scores of fAAUT during test and retest for both patients and clinicians are shown in Table 2. The patient's self-evaluation and clinician's evaluation did not significantly differ between test to retest in both the LHD group (paired $t$-test, $t=-.627, p=.540 ; t=-1.441, p=.170$, for patient's and clinician's evaluation, respectively) and RHD group $(t=-1.229, p=.245 ; t=.457, p=.656$, for patient and clinician's evaluation, respectively). Additionally, there was no difference in self-evaluation error between test to re-test in both groups, even after performance feedback was provided (paired $t$-test, $t=.072, p=.943 ; t=-1.403$, $p=.188$ for LHD and RHD, respectively). The one-sample $t$-test revealed that the self-evaluation errors were not significantly different from zero in the RHD group $(t=-.413, p=.688 ; t=.822, p=.428$ for test and retest, respectively). In contrast, the self-evaluation errors were significantly lower than zero during test $(t=-2.728$, $p=.016)$ and retest $(t=-3.988, p=.001)$ in the LHD group.

\section{The Influence of Stroke Side on Self-evaluation Error}

Next, we conducted a linear mixed model analysis with group (LHD and RHD) and tests (test and re-test) as the fixed factors 
Table 2. Scores of Patient and clinician, and self-evaluation errors in LHD and RHD groups

\begin{tabular}{|c|c|c|c|c|c|c|}
\hline \multirow{2}{*}{$\frac{\text { Group }}{\text { fAAUT }}$} & \multicolumn{3}{|c|}{ LHD $(n=16)$} & \multicolumn{3}{|c|}{$\operatorname{RHD}(n=12)$} \\
\hline & Patient & Clinician & Self-evaluation error & Patient & Clinician & Self-evaluation error \\
\hline Test & $2.29 \pm 1.47^{\mathrm{a}}$ & $3.03 \pm 1.28$ & $-0.74 \pm 1.09 *$ & $2.79 \pm 1.30$ & $2.92 \pm 0.87$ & $-0.13 \pm 1.09$ \\
\hline Retest & $2.40 \pm 1.37$ & $3.16 \pm 1.20$ & $-0.76 \pm 0.76 * * *$ & $3.04 \pm 1.34$ & $2.86 \pm 1.01$ & $0.18 \pm 0.76$ \\
\hline
\end{tabular}

${ }^{\mathrm{a}}$ Mean $\pm \mathrm{SD}$.

$* p<.05$.

$* * * p<.001$ by one-sample $t$-test to confirm that evaluation gap was different from zero.

and each individual as a random factor to analyze the effect of group and clinician feedback on self-evaluation error. The best-fit mixed-effects model included the effect of group and random intercept of patients because the model fit improved by adding group compared with the random intercept of patients alone. (AIC $=131.05$ vs. 126.35, Log-likelihood test, $\chi^{2}=6703, p=.009$ ). Adding tests and the interaction between group and test did not improve the model fit. The best-fit model revealed that participants in the LHD group had a lower self-evaluation error in test and retest, indicating, on average, they underestimated their performance more than patients in the RHD group $\left(t=2.350, \quad p=.019, \quad\right.$ partial $\left.\omega^{2}=.18\right) \quad$ (Figure 2a). Furthermore, we analyzed the frequencies of correct, under-, and overestimation scores in each group across tests. The chi-square test revealed that the number of patients who underestimated their performance was significantly greater in the LHD group than in the RHD group at retest $\left(\chi^{2}=9.049\right.$, $p=.003)$, and marginally greater at test $\left(\chi^{2}=3.114, p=.07\right)$ (Figure 2b; overall, more than $80 \%$ of the LHD group underestimated their performance throughout test and retest). On the contrary, patients with RHD tended to overestimate, especially during retest $\left(\chi^{2}=7.428, p=.006\right)$.

\section{Relationship Between Self-evaluation Error and Impairments and Spontaneous Affected Limb Use}

Overall FM score was not significantly correlated with self-evaluation error in either group $(r=-.022, p=.935$ for LHD and $\rho=.047, p=.884$ for RHD) (Figure 3a). Nor of arm, wrist, and hand sub-items of FM were correlated with self-evaluation error (all $p>.05$ ). Additionally, the use of the more-affected arm was not significantly correlated with self-evaluation error (LHD, $r=-.265, p=.320$ RHD, $r=-.067, p=.835$ ) (Figure 3b). These results indicated that self-evaluation errors occurred in patients with chronic stroke regardless of motor impairment score and more-affected arm use during ADL. Exploratory analyses found no correlations between self-evaluation errors and age, onset months poststroke, or MMSE scores in either group.

\section{DISCUSSION}

This study found hemispheric differences in self-evaluation errors based on video recordings among chronic stroke survivors. Video-based self-observation has several benefits in evaluating performance. For example, individuals have a more generalized awareness of their movement from the third-person perspective (Besharati, Kopelman, Avesani, Moro \& Fotopoulou, 2015), and 'offline' playback can be paused easily frame by frame for participants to evaluate carefully over a relatively long period, compared with the 'online' awareness interviews or questionnaires (Besharati et al., 2015; Fotopoulou et al., 2009). In addition, participants might be more comfortable evaluating past poor performance 'offline', which may prevent a reduction in self-esteem. (Marcel et al., 2004). With the video-based feedback, we found that chronic stroke patients with LHD significantly underestimated their performance, while patients with RHD under-, over-, or correctly estimated their movement performance. These findings are in line with previous research showing the underestimation of LHD (Fowler et al., 2018), and the overestimation of RHD during the bimanual UE tasks in acute-tosubacute phases (Marcel et al., 2004).

One possible explanation for the hemispheric differences in self-evaluation is the frequency of use of the more-affected arm. For example, patients with LHD tended to use their impaired right arm more than patients with RHD who used their impaired left arm, because right-hand preferences exist even after stroke (Mani et al., 2014). Consequently, patients with LHD experienced more errors or unsuccessful movements than those with RHD. In contrast, patients with RHD experienced fewer errors or unsuccessful movements because they did not attempt to move their more-affected left arm (nondominant arm). Repeated negative consequences, such as spilling hot coffee or dropping dishes when using a more-affected arm, might cause disappointment in patients with stroke (Taub et al., 2002; Uswatte \& Taub, 1999), which may lead to performance underestimation. However, patients with RHD use their intact dominant right arm approximately four times more frequently than move their more-affected left arm (Haaland et al., 2012). Therefore, they might not have experienced enough movement attempts to determine their performance levels. This association between frequency of movement and unawareness is also observed during bipedal actions when patients in the acute phase have fewer opportunities to attempt bipedal tasks in the hospital (Marcel et al., 2004).

Another hypothesis to explain self-evaluation errors is the general superiority of the right hand for motor skills, while the left hand is regarded as the weaker counterpart. The ADL 

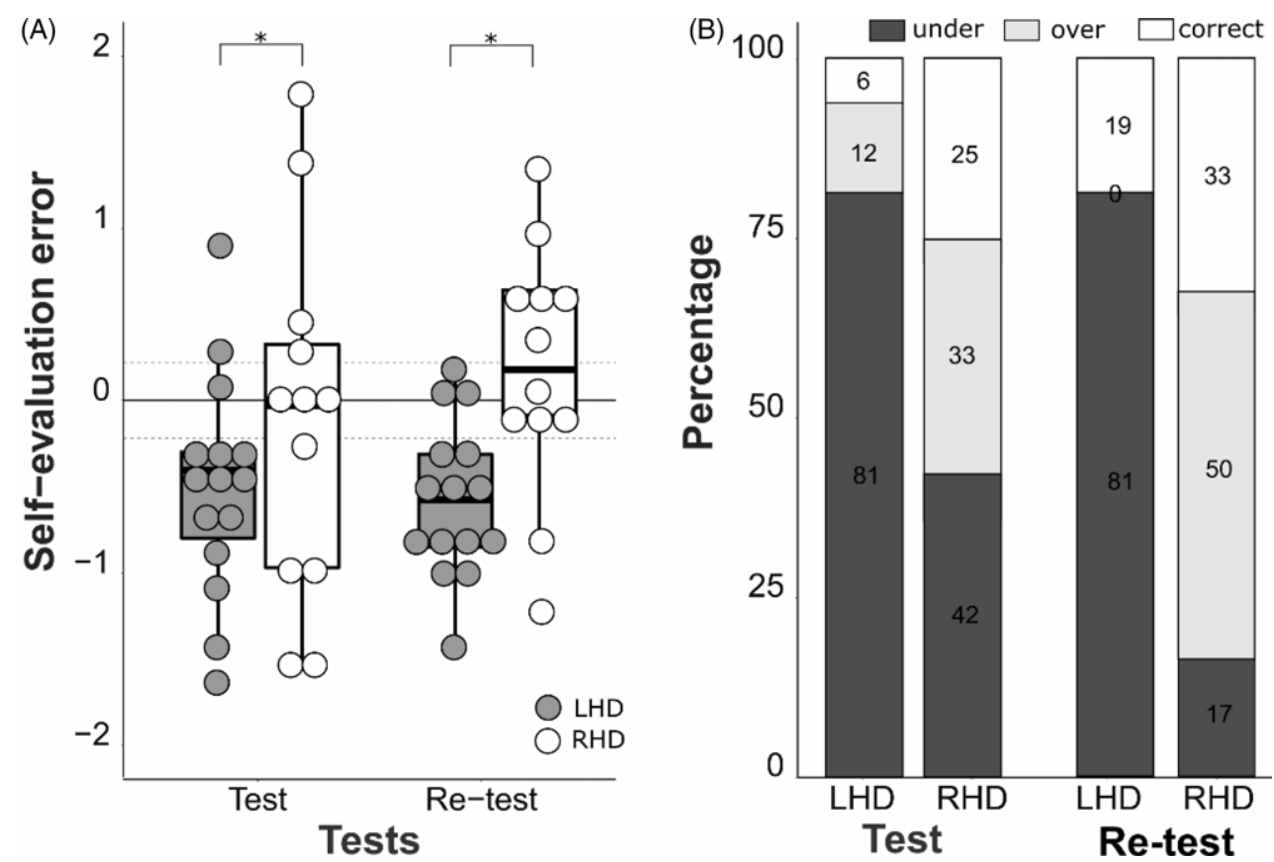

Fig. 2. The self-evaluation errors between LHD and RHD groups during test and retest 2. (A) Self-assessment error in the patients with LHD was lower than in the patients with RHD (mixed-effect model, $t=2.350, p=.019$ ). They consistently underestimated performance, while patients with RHD did not. Self-evaluation errors did not change across tests for both groups $(t=.899, p=.368$ ). (B) Proportion of under-, correct, and overestimation in patients with RHD and LHD. The number of patients underestimated their performance was significantly greater in the LHD group than in the RHD group (chi-square test, $\chi^{2}=3.114, p=.07$ and $\chi^{2}=9.049, p=.003$ for test and re-test, respectively). Overall, more than $80 \%$ of the patients with LHD underestimated while the patients with RHD evenly performed under-, over-, and correct estimations. LHD: left hemisphere damaged group, RHD: right hemisphere damaged group.
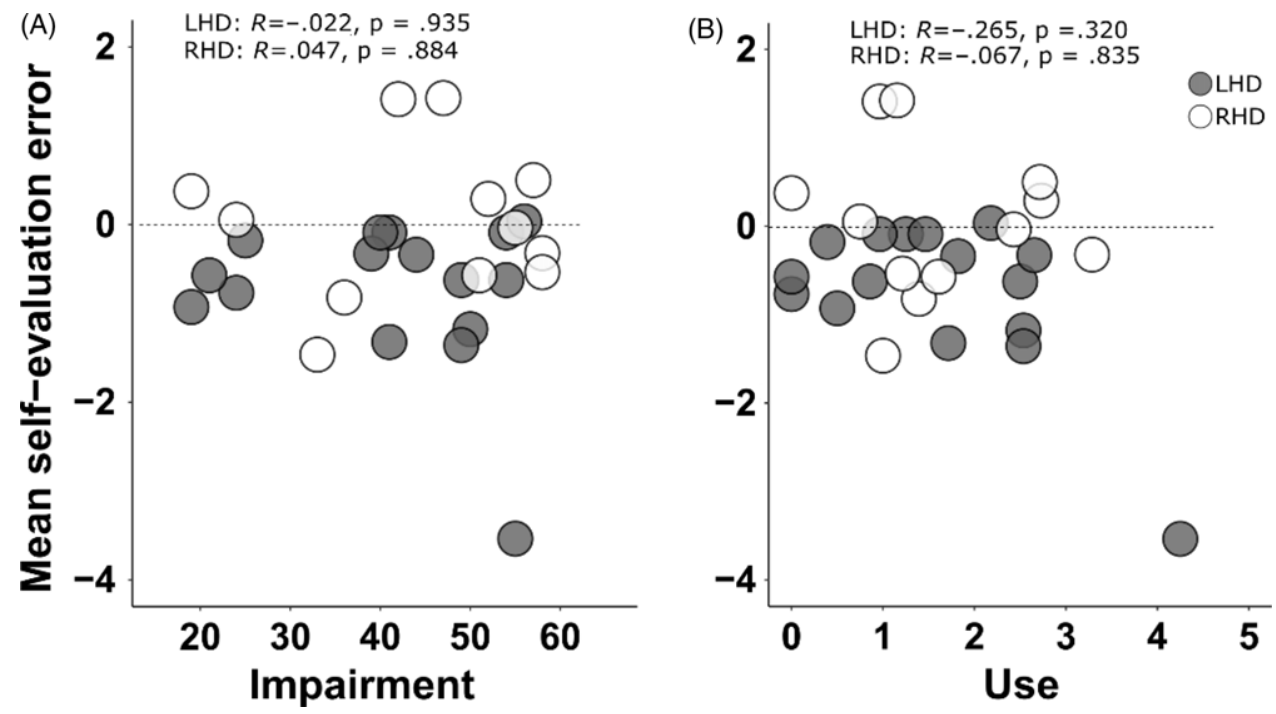

Fig. 3. Correlation plots of self-evaluation error and impairment (A) and use (B). Patients with LHD and RHD did not show an association between self-evaluation error and impairment, as well as between self-evaluation error and spontaneous use of the more-affected arm.

tasks that we used were bimanual tasks requiring equal contribution from both hands (Han et al., 1973; Kim et al., 2019); however, each hand had a specific role in the tasks. For example, holding a paper with the left hand and writing a pen with the right hand. Typically, skilled movements are accurately performed using the dominant right arm (Haaland \& Harrington, 1996; Sainburg, 2002). Consistent with the Dunning-Kruger' effect, such that overestimation is observed in the least skilled arm and hand, and vice versa (Mahmood, 2016; Schlösser, Dunning, Johnson \& Kruger, 2013), patients with LHD who have better motor skills in their dominant right hand are more likely to underestimate their performance. In addition, they might not be satisfied with their performance when compared to 
their abilities before stroke. Consequently, a performance underestimation will occur.

Additionally, under- and overestimation in patients with LHD or RHD are associated with hemispheric asymmetry in the regulation of emotions and attention. The right hemisphere is more likely to process emotional material than the left hemisphere (Wager, Phan, Liberzon \& Taylor, 2003). Patients with RHD often show alexithymia, which is an impairment of decoding, expressing, and awareness of feelings, and an attention deficit (Carson et al., 2000; Spalletta, Ripa, Bria, Caltagirone \& Robinson, 2006). Thus, the patients with RHD in our study might be less concerned about their errors or unsuccessful use of the more-affected arm. In contrast, patients with LHD express catastrophic reactions to their disease and are often in a low mood (Carson et al., 1973; Fowler et al., 2018); therefore, they may have more concerns about their errors and unsuccessful trials made by the more-affected arm in this study. Interestingly, there was no difference in self-evaluation error even after accurate feedback from clinicians (self-evaluation error at test: $-0.74 \pm 1.09$, self-evaluation error at retest: $-0.76 \pm 0.76$, $p>.05$ in LHD, and self-evaluation error at test: $-0.13 \pm 1.09$, self-evaluation error at retest: $0.18 \pm 0.76$, $p>.05$ in RHD). Our participants obtained the clinician's evaluation before they performed the fAAUT on the second day. However, this one-time feedback was not strong enough to change the participants' self-evaluations. Evaluation of the AAUT with 14 different ADLs using the QOM scale was too complex for our participants; therefore, there was a possibility that the patients did not fully understand or accept the one-time feedback from the clinician. Therefore, continuous and repeated reporting of actual scores may be necessary to reduce self-evaluation errors and improve movement self-awareness.

Self-evaluation errors were not correlated with FM-UE or sAAUT scores. Previous studies have shown that actual motor ability is associated with self-evaluation; patients with more severe impairments tend to overestimate their movement and vice versa in acute-to-subacute stroke (Fowler et al., 2018). However, our patients exhibited chronic stroke impairments and ADL tasks in the AAUT focused on bimanual upper extremity movements rather than general gross movements. Therefore, over two-thirds of our participants the LHD group underestimated their movement, leading to no correlation between self-evaluation errors and impairment.

Furthermore, we did not find a significant relationship between self-evaluation error and the actual use of the more-affected arm. This might indicate that correct or overestimation of movements does not necessarily guarantee more use of the more-affected arm. Interestingly, use of the more-affected arm in sAAUT was similar between groups $(1.60 \pm 1.15,1.60 \pm .97 \pm$ for LHD and RHD groups, respectively), while self-evaluation was not. Because AAUT consists of bimanual tasks and is typically tested in a laboratory setting over a relatively short period, arm use might not be fully measured. Other methods, such as an accelerometer, to assess the frequency of use of the more-affected arm over a longer time, may be necessary to monitor movements in the real world and fully elucidate the association between self-estimation error and spontaneous arm use.

This study had several limitations. First, we did not directly use patients' brain imaging data; therefore, we did not know the exact lesion size and location, such as assessing lesions in the anterior and medial prefrontal cortex or medial temporal lobe, which control self-awareness (Berti et al., 2005; Stuss, 1991). Second, we excluded patients with severe motor and cognitive impairments and language and neglect dysfunctions. In addition, three participants in our study experienced recurrent strokes. Because we tried to distinguish the difference between RHD and LHD groups in over-and underestimation of performance, the participants had to have some motor function on their upper extremity (UE). Therefore, we recruited poststroke survivors with mildto-moderate impairment and made a homogenous group.

Finally, all participants reported being right-handed before stroke onset; therefore, our results cannot be generalized to patients who are premorbidly left-handed. Magnetic resonance imaging showing the location of lesions in patients with LHD and RHD should be obtained and voxel-based lesion-symptom mapping between neural substrates and self-evaluation error should be performed in future studies. In addition, patients who are left-handed before stroke onset should be included.

From a clinical perspective, we argue that therapists should be aware of the possibility of patient self-evaluation errors. When patients underestimate their movements, therapists should provide verbal encouragement to let them know that their movements in the more-affected limb are sufficient for executing ADL. In these instances, video recording can be helpful in providing more objective information (Marcel et al., 2004). In addition, patients who overestimate their movements should be supported to notice their limitations so that they can successfully protect themselves from unexpected dangerous situations, including spilling hot coffee or dropping fragile dishes. However, it often upsets patients to see themselves fail while performing a task on a videotape; therefore, a therapeutic alliance should be created with patients before therapy to overcome self-evaluation errors (Prigatano, 2005).

\section{CONCLUSION}

This study investigated the self-evaluation errors in moreaffected arm movements between patients with chronic stroke hemiparesis and clinicians when performing bimanual ADL. Self-evaluation errors existed, but were not correlated with age, FM-UE score, ability to control emotions, or onset months poststroke. Furthermore, the LHD and RHD groups differed in their self-evaluation tendency; patients with LHD underestimated their upper extremity movements, while patients with RHD did not show consistent self-evaluation errors. Taken together, therapists are required to recognize the impaired self-awareness in patients. Furthermore, they 
should develop personalized rehabilitation strategies to overcome any unawareness with respect to the brain lesion side. Using this therapeutic approach, patients can be guided to achieve better results and faster progress in motor re-learning.

\section{AUTHOR CONTRIBUTIONS}

S.C and C.S.K wrote the paper; S.C and C.S.K designed the research; S.C. performed the experiments; S.C, C.S.K, J.W., and K.P analyzed the data; J.W. and K.P edited the paper.

\section{FINANCIAL SUPPORT}

This work was supported by the National Research Foundation of Korea (NRF) grant funded by the Korea government (MSIT) (No. 2017R1C1B5076731).

\section{CONFLICTS OF INTEREST}

The authors have nothing to disclose.

\section{REFERENCES}

Albert M.L. (1973). A simple test of visual neglect. Neurology, 23(6), 658-664. doi: 10.1212/wnl.23.6.658

Berti A., Bottini G., Gandola M., Pia L., Smania N., Stracciari A. .. . et al. (2005). Neuroscience: Shared cortical anatomy for motor awareness and motor control. Science, 309(5733), 488-491. doi: 10.1126/science. 1110625

Besharati S., Kopelman M., Avesani R., Moro V., \& Fotopoulou A. (2015). Another perspective on anosognosia: Self-observation in video replay improves motor awareness. Neuropsychological Rehabilitation, 25(3), 319-352. doi: 10.1080/09602011.2014. 923319

Blumenfeld H. (2002). Neuroanatomy through clinical cases. Sunderland: Sinauer Associates Inc.

Carson A.J., MacHale S., Allen K., Lawrie S.M., Dennis M., House A., \& Sharpe M. (2000). Depression after stroke and lesion location: A systematic review. Lancet, 356(9224), 122-126. doi: 10.1016/S0140-6736(00)02448-X

Cocchini G., Beschin N., Cameron A., Fotopoulou A., \& Della Sala S. (2009). Anosognosia for motor impairment following left brain damage. Neuropsychology, 23(2), 223-230. doi: $10.1037 / \mathrm{a} 0014266$

Cocchini G., Beschin N., \& Della Sala S. (2018). Unawareness for motor impairment and distorted perception of task difficulty. Journal of the International Neuropsychological Society, 24(1), 45-56. doi: 10.1017/S1355617717000662

Cocchini G., Beschin N., Fotopoulou A., \& Della Sala S. (2010). Explicit and implicit anosognosia or upper limb motor impairment. Neuropsychologia, 48(5), 1489-1494. doi: 10. 1016/j.neuropsychologia.2010.01.019

D’Imperio D., Bulgarelli C., Bertagnoli S., Avesani R., \& Moro V. (2017). Modulating anosognosia for hemiplegia: The role of dangerous actions in emergent awareness. Cortex, 92, 187-203. doi: 10.1016/j.cortex.2017.04.009

Della Sala S., Cocchini G., Beschin N., \& Cameron A. (2009). Vata-m: Visual-analogue test assessing Anosognosia for motor impairment. The Clinical Neuropsychologist, 23(3), 406-427. doi: 10.1080/13854040802251393
Duncan P.W., Bode R.K., Lai S.M., \& Perera S. (2003). Rasch analysis of a new stroke-specific outcome scale: The stroke impact scale. Archives of Physical Medicine and Rehabilitation, 84(7), 950-963. doi: 10.1016/S0003-9993(03) 00035-2

Fischer S., Gauggel S., \& Trexler L.E. (2004). Awareness of activity limitations, goal setting and rehabilitation outcome in patients with brain injuries. Brain Injury, 18(6), 547-562. doi: 10.1080/ 02699050310001645793

Fotopoulou A., Pernigo S., Maeda R., Rudd A., \& Kopelman M.A. (2010). Implicit awareness in anosognosia for hemiplegia: Unconscious interference without conscious re-representation. Brain, 133(12), 3564-3577. doi: 10.1093/brain/awq233

Fotopoulou A., Rudd A., Holmes P., \& Kopelman M. (2009). Self-observation reinstates motor awareness in anosognosia for hemiplegia. Neuropsychologia, 47(5), 1256-1260. doi: 10. 1016/j.neuropsychologia.2009.01.018

Fowler E.A., Della Sala S., Hart S.R., \& McIntosh R.D. (2018). Over- and underestimation of motor ability after a stroke: Implications for anosognosia. Neuropsychologia, 119, 191-196. doi: 10.1016/j.neuropsychologia.2018.08.007

Fugl-Meyer A.R., Jääskö L., Leyman I., Olsson S., \& Steglind S. (1975). The post-stroke hemiplegic patient 1. A method for evaluation of physical performance. Scandinavian Journal of Rehabilitation Medicine, 7(1), 13-31. Retrieved from http:// www.ncbi.nlm.nih.gov/pubmed/1135616

Haaland K.Y., \& Harrington D.L. (1996). Hemispheric asymmetry of movement. Current Opinion in Neurobiology, 6(6), 796-800. doi: 10.1016/S0959-4388(96)80030-4

Haaland K.Y., Mutha P.K., Rinehart J.K., Daniels M., Cushnyr B., \& Adair J.C. (2012). Relationship between arm usage and instrumental activities of daily living after unilateral stroke. Archives of Physical Medicine and Rehabilitation, 93(11), 1957-1962. doi: 10.1016/j.apmr.2012.05.011

Han C.E., Kim S., Chen S., Lai Y.-H., Lee J.-Y., Osu R. ...et al. (2013). Quantifying arm nonuse in individuals poststroke. Neurorehabilitation and Neural Repair, 27(5), 439-447. doi: 10.1177/1545968312471904

Hartman-Maeir A., Soroker N., \& Katz N. (2001). Anosognosia for hemiplegia in stroke rehabilitation. Neurorehabilitation and Neural Repair, 15(3), 213-222. doi: 10.1177/15459683 0101500309

Hartman-Maier A., Soroker N., Ring H., \& Katz N. (2002). Awareness of deficits in stroke rehabilitation. Journal of Rehabilitation Medicine, 34(4), 158-164. doi: 10.1080/16501 970213236

Hidaka Y., Han C.E., Wolf S.L., Winstein C.J., \& Schweighofer N. (2012). Use it and improve it or lose it: Interactions between arm function and use in humans post-stroke. PLoS Computational Biology, 8(2), e1002343. doi: 10.1371/journal.pcbi.1002343

Jehkonen M., Laihosalo M., \& Kettunen J. (2006). Anosognosia after stroke: Assessment, occurrence, subtypes and impact on functional outcome reviewed. Acta Neurologica Scandinavica, 114(5), 293-306. doi: 10.1111/j.1600-0404.2006.00723.x

Jenkinson P.M., Preston C., \& Ellis S.J. (2011). Unawareness after stroke: A review and practical guide to understanding, assessing, and managing anosognosia for hemiplegia. Journal of Clinical and Experimental Neuropsychology, 33(10), 1079-1093. doi: 10.1080/13803395.2011.596822

Kim K., Shin Y., Lim M., Jung Y., Oh J., \& Kim S. (2019). Studies on motor activity $\log -28$ and actual amount of use test, actual amount of use test inter-rater reliability in healthy individuals: 
age dependence and handedness. Physical Therapy Korea, 26(2), 24-33. doi: 10.12674/ptk.2019.26.2.024

Kim S., Park H., Han C.E., Winstein C.J., \& Schweighofer N. (2018). Measuring habitual arm use post-stroke with a bilateral time-constrained reaching task. Frontiers in Neurology, CT, doi: 10.3389/fneur.2018.00883

Lang C.E., Strube M.J., Bland M.D., Waddell K.J., Cherry-Allen K.M., Nudo R.J. ... et al. (2016). Dose response of task-specific upper limb training in people at least 6 months poststroke: A phase II, single-blind, randomized, controlled trial. Annals of Neurology, 80(3), 342-354. doi: 10.1002/ana.24734

Lee K., Cheong H.-K., Oh B., \& Hong C. (2009). Comparison of the validity of screening tests for dementia and mild cognitive impairment of the elderly in a community : K-MMSE, MMSE-K, MMSE-KC, and K-HDS. Journal of the Korean Neuropsychiatric Association, 48, 61-69.

Mahmood K. (2016). Do people overestimate their information literacy skills? A systematic review of empirical evidence on the dunning-kruger effect. Communications in Information Literacy, 10(2), 198-213. doi: 10.15760/comminfolit.2016. 10.2.24

Mani S., Przybyla A., Good D.C., Haaland K.Y., \& Sainburg R.L. (2014). Contralesional arm preference depends on hemisphere of damage and target location in unilateral stroke patients. Neurorehabilitation and Neural Repair, 28(6), 584-593. doi: 10.1177/1545968314520720

Marcel A.J., Tegnér R., \& Nimmo-Smith I. (2004). Anosognosia for plegia: Specificity, extension, partiality and disunity of bodily unawareness. Cortex, 40(1), 19-40. doi: 10.1016/S0010-9452 (08)70919-5

Mizuno M. (1991). Neuropsychological characteristics of right hemisphere damage: Investigation by attention tests, concept formation and change test, and self-evaluation task. The Keio Journal of Medicine, 40(4), 221-234. doi: 10.2302/kjm.40.221

Nakase T., Yoshioka S., \& Suzuki A. (2011). Free radical scavenger, edaravone, reduces the lesion size of lacunar infarction in human brain ischemic stroke. BMC Neurology, 11(1), 39. doi: 10.1186/ 1471-2377-11-39

Nurmi Laihosalo M.E., \& Jehkonen M. (2014). Assessing anosognosias after stroke: A review of the methods used and developed over the past 35 years. Cortex, 61, 43-63. doi: 10.1016/j.cortex. 2014.04.008

Orfei M.D., Robinson R.G., Prigatano G.P., Starkstein S., Rüsch N., Bria P.... et al. (2007). Anosognosia for hemiplegia after stroke is a multifaceted phenomenon: A systematic review of the literature. Brain, 130(12), 3075-3090. doi: 10.1093/brain/awm106

Pia L., Neppi-Modona M., Ricci R., \& Berti A. (2004). The anatomy of anosognosia for hemiplegia: A meta-analysis. Cortex, 40(2), 367-377. doi: 10.1016/S0010-9452(08)70131-X

Pia L., Spinazzola L., Rabuffetti M., Ferrarin M., Garbarini F., Piedimonte A. ...et al. (2013). Temporal coupling due to illusory movements in bimanual actions: Evidence from anosognosia for hemiplegia. Cortex, 49(6), 1694-1703. doi: 10.1016/j.cortex. 2012.08.017

Prigatano G.P. (1996). Behavioral limitations TBI patients tend to underestimate: A replication and extension to patients with lateralized cerebral dysfunction. Clinical Neuropsychologist, 10(2), 191-201. doi: 10.1080/13854049608406680

Prigatano G.P. (2005). Disturbances of self-awareness and rehabilitation of patients with traumatic brain injury: A 20-year perspective. Journal of Head Trauma Rehabilitation, 20(1), 19-29. doi: 10.1097/00001199-200501000-00004
Sainburg R.L. (2002). Evidence for a dynamic-dominance hypothesis of handedness. Experimental Brain Research, 142(2), 241-258. doi: 10.1007/s00221-001-0913-8

Schlösser T., Dunning D., Johnson K.L., \& Kruger J. (2013). How unaware are the unskilled? Empirical tests of the, signal extraction, counterexplanation for the Dunning-Kruger effect in self-evaluation of performance. Journal of Economic Psychology, 39, 85-100. doi: 10.1016/j.joep.2013.07.004

See J., Dodakian L., Chou C., Chan V., McKenzie A., Reinkensmeyer D.J., \& Cramer S.C. (2013). A standardized approach to the Fugl-Meyer assessment and its implications for clinical trials. Neurorehabilitation and Neural Repair, 27(8), 732-741. doi: 10.1177/1545968313491000

Sherer M., Hart T., \& Nick T.G. (2003). Measurement of impaired self-awareness after traumatic brain injury: A comparison of the patient competency rating scale and the awareness questionnaire. Brain Injury, 17(1), 25-37. doi: 10.1080/0269905021000010113

Spalletta G., Ripa A., Bria P., Caltagirone C., \& Robinson R.G. (2006). Response of emotional unawareness after stroke to antidepressant treatment. American Journal of Geriatric Psychiatry, 14(3), 220-227. doi: 10.1097/01.JGP.0000194647.72654.a1

Spinazzola L., Pia L., Folegatti A., Marchetti C., \& Berti A. (2008). Modular structure of awareness for sensorimotor disorders: Evidence from anosognosia for hemiplegia and anosognosia for hemianaesthesia. Neuropsychologia, 46(3), 915-926. doi: 10.1016/j.neuropsychologia.2007.12.015

Starkstein S.E., Jorge R.E., \& Robinson R.G. (2010). The frequency, clinical correlates, and mechanism of anosognosia after stroke. Canadian Journal of Psychiatry, 55(6), 355-361. doi: 10.1177/ 070674371005500604

Sterr A., Freivogel S., \& Schmalohr D. (2002). Neurobehavioral aspects of recovery: Assessment of the learned nonuse phenomenon in hemiparetic adolescents. Archives of Physical Medicine and Rehabilitation, 83(12), 1726-1731. doi: 10.1053/apmr.2002.35660

Stuss D. (1991). Disturbances of selfawareness after frontal system. In D.L. Prigatano, \& G.P. Schacter (Eds.), Awareness of deficit after brain injury (pp. 63-84). New york: Oxford University Press.

Taub E., \& Uswatte G. (2004). Constraint-induced movement therapy based on behavioral neuroscience. In R.G. Frank \& T.R. Elliott (Eds.), Handbook of rehabilitation psychology (pp. 475-496). American Psychological Association, doi: 10.1037/10361-022.

Taub E., Uswatte G., \& Elbert T. (2002). New treatments in neurorehabiliation founded on basic research. Nature Reviews Neuroscience, 3(3), 228-236. doi: 10.1038/nrn754

Team R Development Core (2018). R: a language and environment for statistical computing (vol. 2). Vienna, Austria: R Foundation for Statistical Computing. Retrieved from https:// www.R-project.org.

Uswatte G., \& Taub E. (1999). Constraint-induced movement therapy: New approaches to outcome measurement in rehabilitation. In D.T. Struss, G. Winocur \& I.H. Robertson (Eds.), Cognititve neruorehabilitation: A comprehensive approach (pp. 215-229). Cambridge: Cambridge University Press.

Varghese R., \& Winstein C.J. (2020). Relationship between motor capacity of the contralesional and ipsilesional hand depends on the side of stroke in chronic stroke survivors with mild-to-moderate impairment. Frontiers in Neurology, 10, 1-9. doi: 10.3389/fneur.2019.01340

Wager T.D., Phan K.L., Liberzon I., \& Taylor S.F. (2003). Valence, gender, and lateralization of functional brain anatomy in emotion: A meta-analysis of findings from neuroimaging. Neurolmage, 19(3), 513-531. doi: 10.1016/S1053-8119(03)00078-8 